\title{
Vector Theory for the Scattering of TM-polarized Hermite-Gaussian Electromagnetic Beams by a Double Metallic Slit
}

\author{
R. Salazar-Hernández, G. Montiel-González, J. Mulia-Rodríguez, \\ J. Sumaya-Martinez \\ Facultad de Ciencias, Universidad Autónoma del Estado de Mexico, Toluca, Mexico \\ jsm@uaemex.mx
}

\begin{abstract}
We present a rigorous theory for oblique incident Hermite-Gaussian beams, diffracted by two slits of width $\ell$ and separation $\mathrm{d}$, in a thick metallic screen for the case of polarization $\operatorname{TM}(S)$. The far field spectra as a function of several opto-geometrical parameters, wavelength $\lambda$, slit width $\ell$, separation $d$, incidence angle $\theta_{\mathrm{i}}$ and Hermite order $\mathrm{m}$ is analyzed. In the vectorial diffraction region given when $\lambda / \ell>0.2$, where $\ell$ is the incident wavelength and as a function of the separation between slits $\mathrm{d}$; we have numerically analyzed: the far field spectra, the energy diffracted along the incident beam direction $\left(E_{\mathrm{i}}\right)$, and the validity of an approximate diffraction (scalar) property, namely $\mathrm{E}_{\mathrm{i}}=$ $\mathrm{N} \tau / \lambda$.
\end{abstract}

Keywords: diffraction, scattering, double slit.

\section{Introduction}

Currently there are several rigorous theories of diffraction by plane electromagnetic waves (Enriquez et al., 2011) and Gaussian beams (Mata et al, 1993); (Mata et $a l, 1994)$ by two slits in metallic screens of zero thickness. However these theories do not treat with Hermite-Gauss or oblique incidence, nor thick screens of nonzero thickness (Mata et al, 2008).

In this paper we present a novel rigorous theory of diffraction that allows to consider the illumination by Hermite-Gaussian beams at oblique incidence on two slits of width $\ell$ and separation $d$ in screens with infinite conductivity and thickness $h$.

In particular, we analyze the coupling between slits through the numerical study of the diffracted energy along the direction of the incident $\left(E_{i}\right)$ beam energy as a function of the parameter of separation $d$ between the slits. It is revealed the existence of oscillations in the energy $E_{i}$. We also show that in the case of $\operatorname{TM}(\mathrm{S})$ polarization, the energy $E_{i}$ is special because when compared to other diffraction 
patterns. Finally, we show that the scalar property valid at the scalar region $(\lambda / \ell<0.2)$ $E_{i}=N \tau / \lambda$ (Alvarez-Cabanillas, 1995) is not longer valid.

\section{A Vector Theory of Diffraction}

In Fig. 1 we have two slits on a screen of infinite conductivity, and non-zero thickness denoted by $h$. In this screen you have two parallel to the Oz axis, $\ell$ wide and spaced slits $d$. The display is in the gap and impinges perpendicularly on it a HermiteGaussian beam with wavelength $\lambda=2 \pi / k$ and order $m$. We will use the complex representation for the fields and omit the time factor going forward $e^{-i \omega t} . H$ is the magnetic field when you have the TM (magnetic field parallel to the axis Oz) polarization. The $H$ field satisfies the Helmholtz equation (Mata et al, 1994)

$$
\partial^{2} \mathrm{H} / \partial \mathrm{x}^{2}+\partial^{2} \mathrm{H} / \partial \mathrm{y}^{2}+\mathrm{k}^{2} \mathrm{H}=0 .
$$

Denote by $H_{I}$ the solution of Eq (1) in the region I $(y>h / 2)$, expressed by a plane wave expansion:

$$
H_{I}(x, y)=\frac{1}{\sqrt{2 \pi}} \int_{-k}^{k} A(\alpha) e^{i(\alpha x-\beta y)} d \alpha+\frac{1}{\sqrt{2 \pi}} \int_{-k}^{k} B(\alpha) e^{i(\alpha x+\beta y)} d \alpha .
$$

The first integral is identified with the incident beam due to the sign of the $\alpha$ and $\beta$ k-components.

In region II, within the screen, $-h / 2<y<h / 2$ the electromagnetic field will be represented by the following modal series:

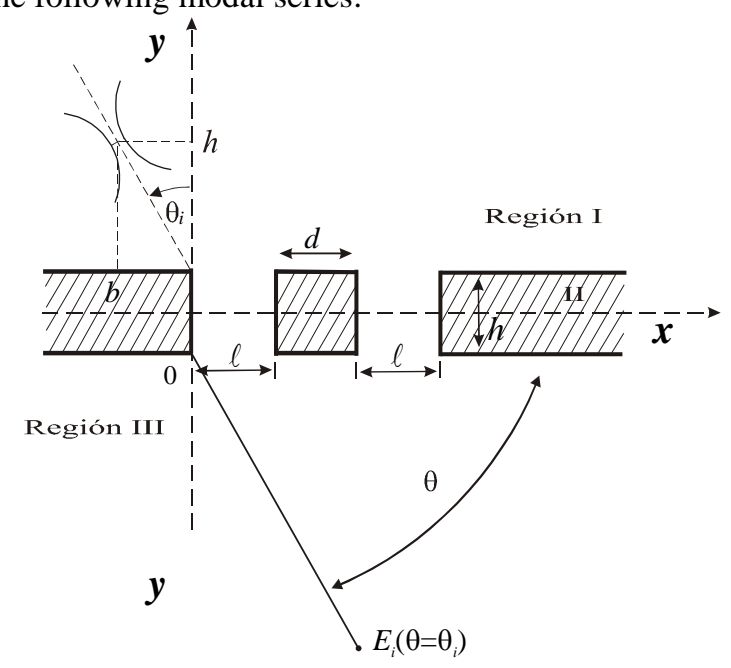

Fig. 1. Our system. Two slits of width $\ell$ and spacing $d$ in an infinitely thick conducting screen $h$. The energy diffracted along the incident direction $\left(E_{i}\right)$ is diffracted in the direction of $\theta($ relative to the axis Oy $)=\theta_{i}($ From the axis Ox $)$. 


$$
H_{I I}(x, y)=\sum_{n=0}^{\infty} a_{n}^{1} \varphi_{n}^{1}(x, y)+\sum_{n=0}^{\infty} a_{n}^{2} \varphi_{n}^{2}(x, y),
$$

where in $i=1,2$ the set $\varphi_{n}^{i}(x)$, are functions whose normal derivative is zero at the walls for the TM polarization.

The diffracted field below the screen, for $y<-h / 2$ (region III), could be expressed as a plane wave expansion too:

$$
H_{I I I}(x, y)=\frac{1}{\sqrt{2 \pi}} \int_{-\infty}^{\infty} C(\alpha) e^{i(\alpha x+\beta y)} d \alpha .
$$

Our goal is to determine the transmitted field (Eq. (4)), for which one needs to determine $C(\alpha)$. Note that $C(\alpha)$ depends on the coefficients $a_{n}^{1}$ and $a_{n}^{2}$ and the incident amplitude $A(\alpha)$. For this, we use the appropriate conditions of continuity, which could be obtained from Maxwell's equations (Alvarez-Cabanillas, 1995). These conditions lead us to the following matrix system, in which the matrix columns $\boldsymbol{a}_{\mathbf{1}}$ and $\boldsymbol{a}_{2}$ are formed respectively by the coefficient $a_{n}^{1}$ and $a_{n}^{2}$.

$$
\begin{aligned}
& M_{11} a_{1}+M_{12} a_{2}=S_{1}, \\
& M_{21} a_{1}+M_{22} a_{2}=S_{2},
\end{aligned}
$$

where $M_{i k}(i, k=1,2)$ are square matrices dependent on the opto-geometrical parameters and $S_{i}(i=1,2)$ are matrices depending only on $A(\alpha)$. The determination of the modal coefficients $a_{n}^{1}$ and $a_{n}^{2}$. allow us to calculate the diffracted field in any region for TM polarization.

\section{Results and Discussion}

Using the complex Poynting vector is possible to obtain the diffracted intensity at the angle $\theta$. For a Hermite-Gaussian beam, the spectral amplitude is (Mata et al, 2008):

$$
\begin{gathered}
\mathrm{A}(\alpha)=\frac{\mathrm{L}}{2} \mathrm{i}^{\mathrm{m}} \mathrm{H}_{\mathrm{m}}\left[-\frac{\mathrm{L}}{2}\left(\alpha \sin \theta_{\mathrm{i}}-\beta \cos \theta_{\mathrm{i}}\right)\right] \times\left[\sin \theta_{\mathrm{i}}+\left(\frac{\alpha}{\beta}\right) \cos \theta_{\mathrm{i}}\right] \mathrm{e}^{(-\mathrm{i} \alpha \mathrm{b})} \times \\
\mathrm{e}^{\left[-\left(\alpha \sin \theta_{\mathrm{i}}-\beta \cos \theta_{\mathrm{i}}\right)^{2} \mathrm{~L}^{2} / 8\right]},
\end{gathered}
$$

where $H_{m}$ is the Hermite polynomial of order $m$. The position of the beam waist is given by the parameter $b$ (see Fig. 1).

In the figures relating to energy diffracted along the direction of the incident beam is $E_{i}\left(\theta=\theta_{i}\right)$ the diffracted angle in the direction of the incident beam, measured from the axis Ox and $\theta_{i}$ is the angle of incident beams to the axis Oy measured. The energy, the diffracted intensity $I(\theta)$ and the transmission coefficient $\tau$ are normalized to the total incident energy $I_{0}$. All parameters normalized opto-geometrical width lof the slots $\ell$, that is, $\ell=1$.

In Figs. 2 and 3 show the diffraction patterns of Hermite-Gaussian beams for the fundamental mode $m=0$ at normal incidence and oblique incidence of $30^{\circ}$; the 
wavelength of the incident beams is $\lambda \ell \ell=0.9$, with extremely wide Gaussian beams $\mathrm{L} / \ell=500 / \sqrt{2}$ and fixed at the position $b / \ell=0.5$, the thickness of the screen is $h / \ell$ $=1$ and the gaps between slits are $d / \ell=0,1,3.5$ and 5 .

The shape of the diffraction patterns for the $m=2$ mode, not shown, is identical to the spectra of FIGS. 2 and 3 (with the same opto-geometrical parameters) except for a scaling factor which provides a lower intensity for this mode, from the respective Hermite polynomial.

From these diffraction patterns we have taken the diffracted energy $E_{i}$ along the direction of the incident beams. Figs. 4 and 5 show the behavior of the $E_{i}$ separation according to $d$ for $m=0$ and 2 modes; opto-geometrical parameters of these figures are the same in Fig. 2 and 3.

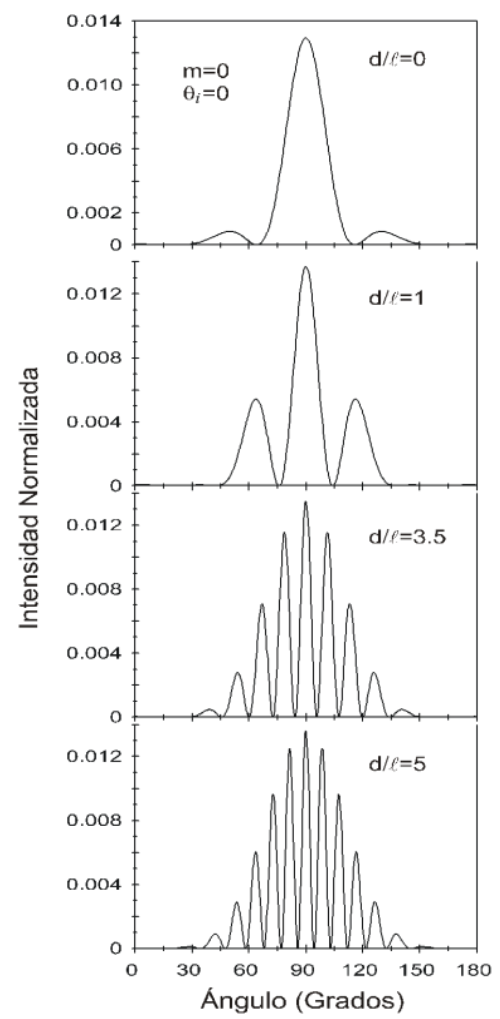

Fig. 2. Diffraction patterns normalized $\left(I(\theta) / I_{0}\right)$ of Hermite-Gaussian beams of $m=0$ normally incident on two slits so. With $\lambda / \ell=0.9, \mathrm{~L} / \ell=500 / \sqrt{2}, h / \ell=1$ and position $b / \ell=0.5$ and for separations $d / \ell=0,1,3.5$ and 5 .

The curves of FIGS. 4 and 5 show the oscillatory behaviors as $E_{i}$ a function of the spacing $d$, in particular for the period is normal incidence to oblique incidence $\lambda$ and the period is $2 \lambda$. 
Vector Theory for the Scattering of TM-polarized Hermite-Gaussian Electromagnetic beams ...

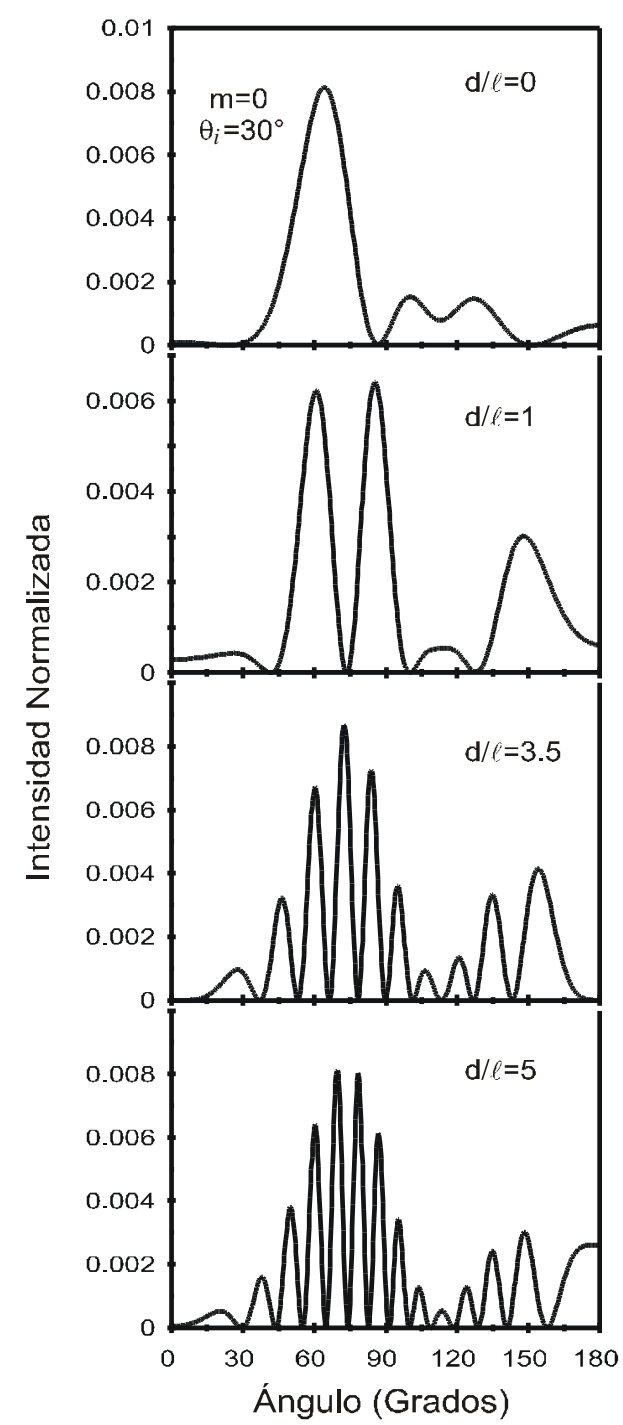

Fig. 3. Standard diffraction patterns $\left(I(\theta) / I_{0}\right)$ Hermite-Gaussian beam for $m=0$ to $30^{\circ}$ obliquely incident on two slits so. Same parameters of Fig.2.

In Fig. 5 has also been drawn in broken lines the $2 \tau / \lambda$ function. As you can see, this function does not overlap with the energy with $E_{i}$ which we can say that the property of diffraction $E_{i}=2 \tau / \lambda$ is not valid in the vector region at least for the separation parameter $d$ and doing extremely wide.

Finally, in Fig. 6 different diffracted energy around the energy is $E_{i}$. The upper curves of Figure 6 correspond to normal incidence for the $m=2$ mode, with the same parameters of Fig. 3; diffracted energies correspond to the angles diffracted $\theta=$ $90^{\circ}, 91^{\circ}, 92^{\circ}$ and $94^{\circ}$. The curves in the lower window of Fig. 6 correspond to 
oblique incidence of $30^{\circ}$, also for mode $m=2$, with the same parameters of Fig. 4 . The diffracted energies shown, corresponding to angles diffracted around of $\theta=60$ - (corresponding to the diffracted energy along the oblique incidence angle $\theta_{i}=$ $30^{\circ}$ ) and for the angles $58^{\circ}, 57^{\circ}$ and $64^{\circ}$.

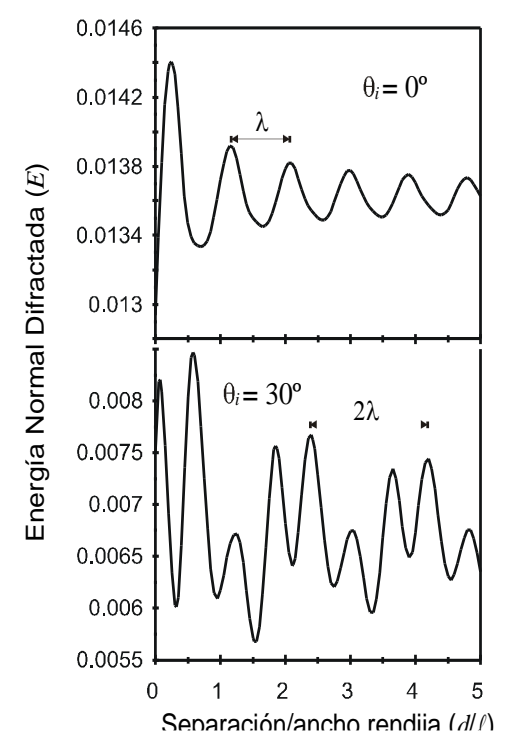

Fig. 4. Energy diffracted in the direction normal to the standard $E_{i}$ to Hermite-Gauss beam, depending on the spacing $d / \ell$ screen. For the fundamental mode $m=0$, at normal incidence and oblique incidence of $30^{\circ}$, with $\lambda / \ell=0.9, \mathrm{~L} / \ell=500 / \sqrt{2}, h / \ell=1, \mathrm{y} \mathrm{b} / \ell=0.5$.

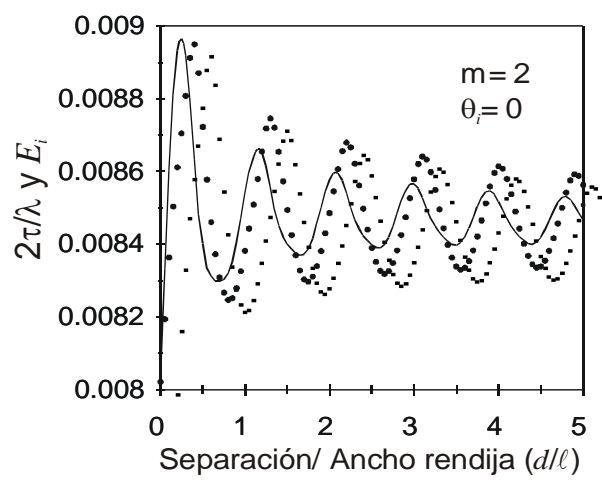

Fig. 5. Energy diffracted in the direction normal to the $E_{i}$ (solid line) Hermite-Gauss beam, thus $m=2$ and $2 \tau / \lambda$ property (dashed line), in function of the spacing $d / \ell$. Same parameters of Fig. 3 .

Energy analyzing energy diffracted $E_{i}$ around for $m=0$ at normal incidence and oblique incidence of $30^{\circ}$ as also carried out (data not shown) found similar patterns 
for mode $m=2$ (see Fig. 6), the energy diffracted around the energy as $E_{i}$ a function of the spacing $d$, decay to zero.

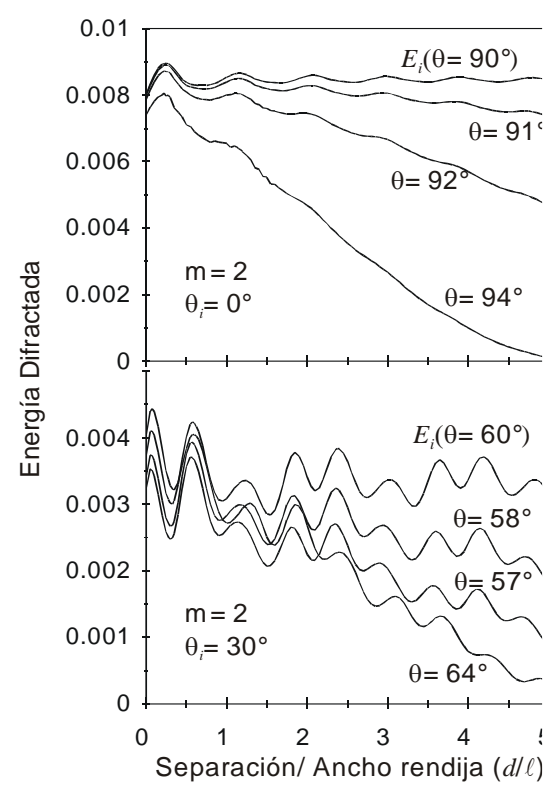

Fig. 6. Energy diffracted around energy $E_{i}(\theta=\theta i)$ Hermite-Gauss beam, for the $m=2$ mode according to the distance between slits $d / \ell$. Same parameters of Fig. 4.

\section{Conclusions}

Present a more rigorous theory of diffraction for the oblique incidence beam HermiteGaussian (HG) on a screen of thickness $h$ with wide slits separating slits $\ell$ and $d$. In the case of $\mathrm{TM}(\mathrm{S})$ polarization and wavelengths in the vector region $\frac{\lambda}{\ell}>0.2$, we have found that the diffracted along the direction of the incident beam energy has oscillations period $\lambda$ as a function of the spacing $d$ for modes $m=0$ and 2 , for the period $2 \lambda$ at $30^{\circ}$ oblique incidence. Finally, we note that the energy $E_{i}$ has special characteristics compared diffracted energies in other directions and found numerically that ownership of scalar diffraction $(\lambda / \ell<0.2)$ given by $E_{i}=2 \tau / \lambda$ is no longer valid in this region $(\lambda / \ell>0.2)$.

\section{References}

1. Enriquez-Leon, G., Martinez-Flores, M. G., Torres-Morales, G., Sumaya-Martinez, J.: Rigorous diffraction of Hermite-Gaussian beams by a double slit. TM polarization. Eight Symposium Optics in Industry (2011) 
2. Mata-Méndez, O., Chávez-Rivas, F.: Estudio teórico y numérico de la difracción en óptica electromagnética, II Teoría rigurosa de la difracción de un haz gaussiano por dos rendijas, Polarización TE. Revista Mexicana de Física, 39(5), pp. 706-721 (1993)

3. Mata-Méndez, O., Álvarez-Cabanillas, M. A.: Estudio teórico y numérico de la difracción en óptica electromagnética, III Teoría rigurosa de la difracción de un haz gaussiano por dos rendijas, Polarización TM. Revista Mexicana de Física, 40(6), pp. 846-856 (1994)

4. Mata-Méndez, O., Chávez-Rivas, F., Ortiz-Acebedo, A.: Diffraction of hermite-gaussian beams by Ronchi and aperiodic rulings. Revista Mexicana de Física, 54(1), pp. 35-4 (2008)

5. Álvarez-Cabanillas, M. A.: Tratamiento riguroso para la difracción de un haz HermiteGauss a través de N-rendijas. ESFM-IPN, Tesis Doctoral (1995) 